\title{
BRIEF NOTES ON TOBACCO USE IN AGED POPULATION
}

Turkish Journal of Geriatrics

DOI: 10.31086/tjgeri.2020.149

2020; 23(2): 153-156

\section{- Dilek ASLAN ${ }^{1}$ iD \\ - Meltem ŞENGELEN ${ }^{1}$ (D) \\ - Yeşim Gökçe KUTSAL ${ }^{2}$ (D)}

CORRESPONDANCE

Dilek ASLAN

Hacettepe University, Faculty of Medicine,

Department of Public Health, Ankara, TURKEY

Phone: +903123051590

e-mail: diaslan@hacettepe.edu.tr

Received: April 17, 2020

Accepted: April 19, 2020

${ }^{1}$ Hacettepe University, Faculty of Medicine, Department of Public Health, Ankara, TURKEY

2 Hacettepe University, Faculty of Medicine, Department of Physical Medicine and Rehabilitation and Hacettepe University Geriatric Sciences Research and Application Center (GEBAM), Ankara, TURKEY
Tobacco use is a lethal addiction which is very prevalent in almost all ages. However, prevalence rates decrease in aged population. People usually start tobacco in younger ages and due to a couple of reasons, prevalence decreases in aged population. One major reason may be the fact that tobacco users die before they reach to older ages. Another fact may be the higher quit rates due to any disease/health problem when one ages. Non-communicable diseases are prevalent in older age and tobacco consumption is closely connected with majority of these prevalent conditions. Lung cancer, COPD (chronic obstructive pulmonary disease), heart disease, stroke, diabetes, vision loss due to cataracts and age-related macular degeneration, and almost over ten types of cancer, including colon, cervix, liver, stomach and pancreatic cancer, are examples. Deepa et al. stated that, in older adults, both current and past cigarette smoking increased the risk of heart failure (1). And in current smokers, this risk was high irrespective of packyears of exposure, whereas in past smokers there was a dose-effect association (2).

Healthy aging is a goal for almost all societies since 2000s defined very clearly in the Madrid International Plan in 2002. In this regard, mental and social wellbeing should be focuses as well as the physical component of health as defined by the World Health Organization. Mental and social wellbeing needs a healthy cognitive system in older ages and risky behaviors including smoking which is among preventable threats. Moreover, cognitive system is progressively affected contributing to a decline in cognitive function as early as middle-age. The impact increases with age and amount smoked, although quitting can reduce the risk and harm caused. Hazards are in a wide range. For example, musculoskeletal system is also progressively effected and the damage has been observed to start as early as the late teens and increases with age but both the risk and harm can be partially reversed by quitting. Smoking has a negative impact on bone mineral density, associated with more bone fractures and slower healing, also smokers and exsmokers experience more pain in the back, neck and legs causing disability in performing activities of daily living and maintain quality of life $(3,4)$. 
Heavy smoking was also found to be associated with higher prevalence and severity of periodontitis (5). Alveolar bone loss and implant failure, increased joint disease, poor functional outcomes, and poor therapeutic response was detected by the researches and the writers also found evidence of adverse effects on muscles, tendons, cartilage, and ligaments regarding the locomotor system (6).

Frailty is an emerging gerontological concept describing a multidimensional syndrome characterized by loss of physiologic reserves, which leads to increased vulnerability to age-related diseases and functional impairment. It has been shown that; frail people are at higher risk of agerelated diseases and higher late-life mortality. A systematically research was planned to investigate the association of tobacco smoking with frailty in a population-based cohort of older adults and the authors stated they observed that current smoking, cumulative smoking exposure (packyears), and time after smoking cessation (years) were significantly associated with the frailty index, which is accumulation of deficits as a proxy measure of aging. This study suggested that epigenetic alterations could play a role in smoking-associated development of frailty (7).

Passive smoking also causes mortality and morbidity starting from the very young ages. In some cases, burden may affect elders worse. For example, disaster situations may have the potential to start tobacco in other age groups (8). Or widowhood may be another risk factor as highlighted by Perkins et al in 2018 (9).

There is only one and exact solution to prevent all these risks and unwanted outcomes which is very simple in theory: "tobacco free life". Unfortunately, addiction is not easily avoided and comprehensive tobacco control policies structured for all ages is crucial. Tobacco control refers "not to start to smoke", "prevention from the risks of passive smoking" and "quitting". Aged population generally need quitting services rather than the other two components. Nevertheless, there are factors which may complicate the course mentally and practically although it is a fortunate to know that quitting at any age can provide health and well-being for older people. When a 60 years of age person quits, about three years of life expectancy will be gained. If a patient with a life-threatening disease decides to quit, complications are expected to decrease. For instance, having another heart attack risk is expected to decrease by $50 \%$ among people who quit smoking after a heart attack (10). Also, continuing smoking after cancer diagnosis has been shown to reduce treatment efficacy and thus survival with deterioration in quality of life. Therefore it is crucial to recommend quitting to a cancer patient if $s($ he) smokes (11).

To achieve success in quitting at older ages, we should change a number of myths on the issue at older ages as such myths have the potential to make aged people refrain from quitting tobacco (12). Incorrect information and opinions are needed to be changed systematically by spreading the evidence based content written below (13):

a. Tobacco has no benefit

b. Tobacco smoke does not improve mood

c. Cessation produces significant benefits

d. Reducing the number of cigarettes does not work

When smoking is prevented, both increase in life expectancy at birth and improvement of quality of life are expected. Lei MK et al. conducted regression analyses of methylation data from 22 subjects, as they entered and exited inpatient substance use treatment, to determine change in biological age, as indicated by the deviation of their methylomic age from chronological age across two time points (14). And the results showed that; successful smoking cessation makes patients appear biologically younger than they were at baseline, and to do so quite rapidly. In today's youth driven society, the 
researchers observations may serve as a powerful impetus for some to quit smoking and clinicians counseling patients should consider whether the addition of this information may be useful in persuading patients to quit smoking.

It is clear that, precautions at the earliest ages will provide maximum benefit. In this regard, lifelong smoke-free approach may be a good way to choose at the highest level. There is another important aspect of tobacco-free life in older ages. Most of the aged people are grandparents, they have probably grandchildren which gives them another responsibility to be good role models for their younger generations. Role model identity of older

\section{REFERENCES}

1. Deepa MG, Kalogeropoulos AP, Georgiopoulou W, et al. Cigarette Smoking Exposure and Heart Failure Risk in Older Adults: The Health, Aging, and Body Composition Study. Am Heart J 2012;164(2): 236-42. (PMID: 22877810).

2. Gigante AF, Martino T, lliceto G, Defazio C. Smoking and age-at-onset of both motor and non-motor symptoms in Parkinson's disease. Parkinsonism Relat Disord 2017; 45:94-96. (PMID: 28988683).

3. Murray R. A report commissioned by Public Health England: The role of smoking in the progressive decline of the body's major systems. United Kingdom, 2014. [Internet] Available from:https://assets. publishing.service.gov.uk/government/uploads/ system/uploads/attachment_data/file/774658/The_ role_of_smoking_in_the_progressive_decline_of_ the_bodys_major_systems.pdf. Accessed: 20.3.2020.

4. Abate M, Vanni D, Pantalone A, Salini V. Cigarette smoking and musculoskeletal disorders. Muscles Ligaments Tendons J 2013; 3(2): 63-9. (PMID: 23888288).

5. Khan S, Khalid T, Awan KH. Chronic periodontitis and smoking: Prevalence and dose-response relationship. Saudi Med J 2016;37(8):889-94. (PMID:27464867).

6. Bashaireh M A, Haddad LG, Weaver M, et al. The ages are defined in the literature. Non-smoker role models are chosen by the younger generations (15).

One more fact should be highlighted before concluding. Smoking itself facilitates aging. Shorter telomeres among smokers are found when compared to the non-smokers. This finding highlights the link tobacco smoke exposure and ageing-related disease (16).

Global struggle with tobacco usage frequently focuses on younger ages which can be understandable to basically beat back the tobacco companies' manipulations. However, aged people should not be forgotten in this struggle as we have many promising data on tobacco-free lives.
Effect of Tobacco Smoking on Musculoskeletal Health: A Systematic Review. J Environ Public Health 2018;4184190. [Internet] Available from: https://www. ncbi.nlm.nih.gov/pmc/articles/PMC6077562/pdf/ JEPH2018-4184190.pdf. Accessed: 20.3.2020.

7. Gao X, Zhang Y, Saum KU, et al. Tobacco smoking and smoking-related DNA methylation are associated with the development of frailty among older adults. Epigenetics 2017;12(2):149-56. (PMID: 28001461).

8. Koyama S, Tabuchi T, Aida J, Osaka K, Miyashiro I. (n.d.). Determinants of increased tobacco consumption following a major disaster. Disaster Med Public Health Prep 2020:1-5. (PMID: 32183911).

9. Perkins JM, Lee HY, Lee JK, et al. Widowhood and alcohol, tobacco, and other drug use among older adults in India. J Gerontol B Psychol Sci Soc Sci 2018;73(4): 666-674.(PMID: 28329813).

10. Health benefits of smoking cessation. [Internet] https://www.who.int/news-room/q-a-detail/healthbenefits-of-smoking-cessation. Accessed 23.3.2020.

11. Florou AN, Gkiozos IC, Tsagouli SK, Souliotis KN, Syrigos KN. Clinical significance of smoking cessation in subjects with cancer: a 30-year review. Respir Care 2014;59(12):1924-36. (PMID:25185148).

12. Brown LM. Tobacco myths: the older adult 
perspective. J Gerontol Nurs 2015;41(5):9-13. (PMID: 25642695).

13. Cataldo JK. Clinical Implications of Smoking and Aging: Breaking Through the Barriers. J Gerontol Nurs 2007;33(8):32-41. (PMID: 17718376).

14. Lei MK, Beach SRH, Dogan MV, Philibert RA. A Pilot Investigation of the Impact of Smoking Cessation on Biological Age. Am J Addict 2017;26(2):129-35. (PMID: 28106943).

15. Jopp DS, Jung S, Damarin AK, Mirpuri S, Spini D. Who is your successful aging role model? J Gerontol B Psychol Sci Soc Sci 2017;72(2):237-47. (PMID:27803026).

16. Astuti $Y$, Wardhana A, Watkins J, Wulaningsih W; PILAR Research Network. Cigarette smoking and telomere length: A systematic review of 84 studies and meta-analysis. Environ Res 2017;158: 480-9. (PMID: 28704792). 